\section{Summary}

Radiotherapy is the use of some ionising radiations in medicine to produce a biological effect, either in the form of particles or waves of sufficient energy to produce the effect desired. These radiations are obtained from radioactive elements undergoing spontaneous disintegration (e.g. radium or radon); from elements previously excited which, when reverting to their normal state, give off the energy acquired (e.g. radioactive phosphorus, or strontium) from X-ray tubes where metals (e.g. tungsten) are bombarded with electrons; or from apparatus either designed to accelerate heavy particles and produce a beam of neutrons (Cyclotron) or to accelerate electrons, which may be used directly, but more probably to produce very high-voltage $\mathrm{X}$-rays (Betatron). The absorption of energy from these radiations results in intense electronic activity in the tissues.

The amount of damage done depends on the amount and distribution of the energy absorbed, the rate of absorption, the intervals between exposures, the total treatment period, the sensitivity of the cells, and the nature and state of the tumour bed. A wide variety of apparatus is necessary for the efficient treatment of all types of case. The results of treatment with early accessible cancer are excellent and with deep-seated cancer are improving, and may be expected to improve considerably in the future. Earlier diagnosis and treatment and more knowledge of the biological effects are now more urgently needed than technical advances on existing lines.

\section{Acknowledgement}

This paper is based on a short series of lectures given to medical students at the Royal Free Hospital. I am indebted to Dr. P. C. Koller and to Mr. L. F. Lamerton for the helpful advice they gave me in their preparation.

\title{
THE TREATMENT OF SUPPURATIVE OTITIS MEDIA*
}

\section{By S. E. Birdsall, F.r.c.s.}

My subject deals with suppurative otitis media, acute or chronic, and from the clinical aspect it comprises the majority of patients who present themselves to the practitioner with discharging ears.

It is the practitioner's first duty to decide whether the discharge is from the external meatus or from the middle-ear, and it is obvious that no treatment can logically be prescribed until this distinct point has been settled.

It is an unfortunate fact that the present medical curriculum provides inadequate instruction in many so-called special subjects. Any general practitioner is certain to meet in his practice with many patients suffering from otorrhoea in the course of a year, and they will certainly be greater in number than those whom he finds with appendicitis or carcinoma of the alimentary canal, yet in his studentship he will have gained very scanty knowledge of the common condition of otitis media, compared with his intimate knowledge of the far rarer abdominal diseases.

Confronted with a patient who has a discharging ear, it is essential to know the origin of the discharge; is the diagnosis one of otitis externa or of otitis media? The distinction may be difficult. The following points may be helpful.

* Based on a lecture at The Memorial Hospital, S.E.r 8, on 9.3 .46 .
First.-In otitis externa, tenderness is marked, and pain may be slight. In otitis media, pain may be very severe, and tenderness of the pinna is invariably absent. Mastoid tenderness may be present in either condition.

Secondly.-The character of the discharge affords valuable evidence. In amount, it is more copious in otitis media. All discharge must first be removed by mopping or syringing. If it rapidly reappears, then we are probably dealing with otitis media, in which the secretion in the meatus is the overflow from an infected cavity lined with mucous membrane. In otitis externa, the secretion is derived from the skin lining the meatus, and it will not be replaced in a shorter time than an hour. If the discharge contains mucus, then it must come from the middle-ear as there are no mucous glands in any skin. The discharge in otitis externa is often bright yellow, as though stained with flavine.

Thirdly.-The meatus is usually bealthy in otitis media. In otitis externa there is usually some stenosis and the meatus may be very narrow owing to swelling of the lining skin, which either itches or is acutely tender.

Fourthly.-In a disease affecting the ear, no examination is complete unless the hearing be tested. It is essential first to clean the meatus of discharge. In otitis externa the hearing will be normal or but slightly diminished. In otitis 
media there must always be a considerable hearing loss.

\section{Treatment of Suppurative Otitis Media}

Aural discharge has been during the two great wars the commonest disability rendering a recruit unfit for active service.

Its enormous importance in the health of the community is significantly recognised by the insurance companies, none of whom will contract a life assurance policy with one who suffers from otorrhoea. Such a state of affairs must mean either that we are dealing with a disease of obscure or unknown etiology, or that our treatment is bad. The former is not true, and we must conclude that in this condition we are not treating our patients properly. I have studied this subject closely for twelve years, having been discouraged and distressed by the vast numbers of out-patients who were attending the Aural Department of a great hospital weekly, monthly and yearly, with no change in their condition. It seemed that the treatment they were receiving was at least valueless and possibly harmful.

Not knowing the correct treatment, I at least became well acquainted with the useless or bad methods, and this was a great step forward.

\section{The Origin of Otorrhoea.}

It is essential to examine the disease from its onset. An acute otitis media leads to an empyema of the middle-ear. The increasing pressure of exudate leads to a bulging of the drum-membrane. At this stage a myringotomy should be performed. It shortens the duration of a painful illness, the membrane heals perfectly, the hearing is restored, and the discharge ceases in from one to ten days. It is very rarely that mastoiditis or chronic otorrhoea follows. Myringotomy at an early date is the most certain preventive measure against chronic otitis media. Failing myringotomy the membrane is spontaneously ruptured by pressurenecrosis, leading to an open fistula. The invariable additional factor leading to chronic otorrhoea is now facilitated - and this factor is secondary infection, which is the prime cause of chronicity in all surgical diseases.

\section{Factors Conducive to Secondary Infection.}

This cardinal factor of secondary infection is now reinforced by the treatment given. The meatus itself is the source of the secondary infection and it is obviously wrong to plug it with cottonwool. It is also wrong to instil carbolic acid and glycerin, which will produce a chronic dermatitis and which cannot possibly have any therapeutic effect on the underlying middle-ear disease. There is no pyogenic infection in the body in which the application of 4 per cent phenol to the infected tissue would be regarded as anything but malpraxis. The use of hydrogen peroxide is also to be condemned, as it causes maceration of the skin of the deep meatus, and it cannot have any bactericidal value in the middle-ear, which it cannot reach.

\section{Rational Treatment.}

In a case of acute suppurative otitis media before rupture of the drum-membrane has occurred, we are dealing with a pure infection due to streptococci or pneumococci in the vast majority of cases. If we can prevent secondary infection, the disease will usually resolve in from one to ten days.

How are we to prevent secondary infection? The obvious answer is to keep the meatus surgically clean. This is done by preventing accumulation of discharge, and maintaining the health of its lining skin. The former is best attained by syringing with a sterile non-irritant fluid, such as biniodide of mercury I in 4,000. Mopping with sterile wool is open to the objection that it requires a skilled surgeon, and that even in his hands there is no means of removing secretions pushed in front of his cotton-wool pledget on each application. After the syringing, spirit drops are used at a strength of 25 per cent merely in order to dry the skin surface, and not with the object of killing bacteria.

This treatment should be carried out at least twice daily and the meatus should be left free of any foreign body however sterile. If in spite of this routine the discharge persists for three weeks, there must be a reason for its persistence.

\section{Persistent Otorrhoea.}

If a case of acute suppurative otitis media be treated correctly, the discharge should cease within I4 days. If the otorrhoea be unchanged after this period, it is essential to discover the reason. The factor leading to non-resolution is often discovered on careful examination of the drum-membrane. It is often found that the region surrounding the perforation is occupied by a small bright-red polyp, which is a herniation of granulation-tissue or swollen mucous membrane, which acts as a ball-valve and interferes with drainage of mucopus from the middle-ear. If this be removed or destroyed by the application of chromic acid on a probe, the otorrhoea may be cured dramatically in one or two days, even in cases where it has resisted treatment for months.

The discharge may be persistent and profuse, and it becomes obvious that its volume is greater than could possibly arise from the mucosa of the middleear alone. It is probable in such a case that the mastoid cells are infected and an X-ray examination will confirm the diagnosis. The correct procedure will be to obliterate the infected area by a simple 
cortical mastoid operation, which should be performed without delay, as it offers the only chance of healing of the drum-membrane and restoration of good or perfect hearing.

Persistent otorrhoea may be caused by an untreated neighbouring infected area, such as an antral empyema, infected adenoids or tonsils, or bad teeth. These must be sought, and if found eradicated.

Very rarely indeed, tuberculosis affects the middle-ear and leads to persistent discharge.

\section{Chronic Otorrhoea.}

In a great number of cases, the institution of rational methods of treatment will have most dramatically favourable results.

It may, however, be impossible to promise a dry ear for this reason. There may be a large perforation which can never heal, much of the drummembrane having been destroyed. In such a case we are really dealing with a fistula, connecting the nasopharynx with the exterior, and it is obvious that a fistula leading to a cavity secreting mucus cannot be dry. If we can ensure that the discharge is one of mucus only, we must be content. If the discharge continue purulent and offensive, then we are dealing with an infection that involves not only mucous membrane, but also bone.

A radical mastoid operation will be indicated if the continued purulent discharge indicates a dangerous osteitis. The operation will have as its primary aim the eradication of bone disease that endangers life, and it is important to realise that it is not performed in order to terminate aural discharge.

\title{
MICROCRYSTALLINE SULPHATHIAZOLE IN ABDOMINAL SURGERY
}

\author{
A PRELIMINARY REPORT \\ By R. S. Ninian, F.R.C.s. \\ (Surgeon-in-Charge, Wrexham Emergency Hospital.)
}

In I942, L. A. Chambers, of Philadelphia, and his colleagues ${ }^{1}$ reported favourable results with the local use of 20 per cent microcrystalline suspension in I9 abdominal cases ( 2 exploratory laparotomies, I enterostomy, 3 gastric resections, 7 operations for carcinoma of the large bowel or rectum, I acute pancreatitis, 4 acute appendicitis, I intestinal fistula). They were particularly impressed by the fact that this suspension had very little tendency to "cake" or form insoluble masses in the peritoneal cavity.

Two years later J. T. Chesterman, ${ }^{2}$ of Sheffield, compared the mortality-rate in patients with acute appendicitis and acute diffuse peritonitis treated with I5 per cent microcrystalline sulphathiazole suspension (introduced into the peritoneal cavity) with that in similar patients in whom no sulphonamide drugs were administered. In the first series there was I death among 20 patients, or 5 per cent mortality; in the second there were 15 deaths among 75 cases, or 20 per cent mortality. Chesterman regards intraperitoneal microcrystalline sulphathiazole as of value in the treatment of diffuse peritonitis, though he admits that some of the bacteria causing this condition are sulphonamideresistant. It is of interest to note that there was no evidence of "caking" in the one abdomen which was reopened on the 128th post-operative day.

My series of 9 abdominal cases here tabulated is a small one and may thus be open to criticism, but it presents several valuable features justifying the publication of a preliminary report. As the result of my initial trials I am convinced that microcrystalline sulphathiazole in suspension form is extremely useful in the prevention and treatment of intraperitoneal infections. Furthermore, the dispensing with drainage in local peritonitis and even abscess formation is a great advantage in acute abdominal surgery. An effective local concentration of sulphathiazole is more easily obtained with this fluid preparation than with dry powder.

Of my 9 cases, 4 were acute perforated appendicitis, I appendix abscess, I purulent cholecystitis, I perinephric abscess and tuberculous kidney, I intussusception, and I acute ileal mesenteric adenitis. The operative findings and the results obtained are given in the accompanying table.

\section{REFERENCES}

I. CHAMBERS, L. A., et al. (1942), J. Amer. Med. Ass., 119, 324. 2. CHESTERMAN, J. T. (1944), Lancet, 2, 407. 